Fonoaudiologia Baseada

em Evidências

Evidence-based Speech-Language

Pathology and Audiology

Claudia Regina Furquim de Andrade ${ }^{1}$

Fabiola Staróbole Juste ${ }^{1}$

Descritores

Fonoaudiologia

Gagueira

Equipamentos e provisões

Audição

Keywords

Speech, language and hearing sciences Stuttering

Equipment and supplies Hearing

Endereço para correspondência: Claudia Regina Furquim de Andrade R. Cipotânea, 51, Cidade Universitária, São Paulo (SP), Brasil, CEP: 05360-160.

E-mail: clauan@usp.br

Recebido em: 1/3/2011

Aceito em: 4/5/2011

\section{Análise sistemática da efetividade do uso da alteração do feedback auditivo para a redução da gagueira}

\author{
Systematic review of delayed auditory feedback \\ effectiveness for stuttering reduction
}

RESUMO

Objetivo: Realizar uma revisão sistemática de pesquisas relacionadas aos efeitos da alteração do feedback auditivo sobre a fluência da fala em pessoas com gagueira. Estratégia de pesquisa: Foram seguidos os preceitos do Cochrane Handbook: formulação da pergunta inicial (tema a ser pesquisado), localização e seleção dos estudos (base de dados PubMed) e compatibilização entre os pesquisadores (visando minimizar possíveis perdas de citações). Critérios de seleção: Foram excluídas: citações em línguas que não fossem o Inglês, citações que não permitiram o acesso ao texto completo, citações repetidas por sobreposição das palavraschave utilizadas, pesquisas realizadas exclusivamente com indivíduos fluentes; estudos de caso; revisões de literatura; cartas ao editor e textos que não se relacionavam diretamente ao tema. Desta forma, foram analisados os textos que efetivamente se relacionavam às pesquisas de tratamento com delayed auditory feedback (DAF) e o frequency-altered feedback (FAF). Análise dos dados: Os dados foram analisados quanto aos indicadores da pesquisa e quanto aos marcadores de qualidade dos estudos. Resultados: Os resultados indicaram que o uso dos dispositivos de alteração do feedback auditivo na redução do número de eventos de gagueira ainda não tem suporte robusto em sua aplicabilidade. A variabilidade de método não permite uma resposta ou uma tendência de resposta que possa ser considerada consistente sobre a eficácia do dispositivo. Conclusão: Embora as limitações apontadas nos estudos impeçam generalizações sobre a eficácia do uso do dispositivo para a redução da gagueira, essas mesmas limitações são recursos importantes para o planejamento de pesquisas futuras.

\begin{abstract}
Purpose: To perform a systematic review of studies related to the effects of delayed auditory feedback on speech fluency in individuals who stutter. Research strategy: Concepts of the Cochrane Handbook were followed: formulation of initial question (theme to be reviewed), location and selection of studies (PubMed database) and compatibilization among researchers (aiming to minimize possible citation losses). Selection criteria: The following were excluded: citations in languages other than English, citations that did not allow access to full text, repeated citations due to the overlap of keywords, studies developed exclusively with fluent individuals, case reports, reviews of the literature, letters to the editor, and texts that were not directly related to the theme. Hence, texts that were related to treatment with delayed auditory feedback (DAF) and frequency-altered feedback (FAF) were analyzed. Data analysis: Data were analyzed according to research indicators and according to study quality markers. Results: The results indicated that the use of altered auditory feedback devices for the reduction of stuttering events still do not have robust support for their applicability. Methodological variability does not allow a consistent answer, or a trend about the effectiveness of the device, to be drawn. Conclusion: Although the limitations in the studies prevent generalizations about the effectiveness of the device for the reduction of stuttering, these same limitations are important resources for future research planning.
\end{abstract}

Trabalho realizado no Departamento de Fisioterapia, Fonoaudiologia e Terapia Ocupacional da Faculdade de Medicina, Universidade de São Paulo - USP - São Paulo (SP), Brasil.

(1) Departamento de Fisioterapia, Fonoaudiologia e Terapia Ocupacional da Faculdade de Medicina, Universidade de São Paulo - USP - São Paulo (SP), Brasil. 


\section{INTRODUÇÃO}

Há mais de 50 anos, diferentes estudos têm demonstrado que as alterações no feedback auditivo (AFA) podem diminuir o número de eventos de gagueira ${ }^{(1)}$. O termo AFA pode ser definido como um conjunto de condições que envolvem, eletronicamente, a alteração do sinal de fala, em que o falante percebe a própria voz de forma modificada. As formas mais frequentes de AFA são o delayed auditory feedback (DAF) e o frequency-altered feedback (FAF).

O DAF ocorre quando o retorno auditivo da voz é atrasado numa variação usualmente entre 50 a $100 \mathrm{~ms}$. Com esse recurso, o falante ouve a própria voz como efeito de coro. O FAF referese à variação da frequência da voz do falante, usualmente entre $1 / 4$ a 1 oitava, acima ou abaixo. Com esse recurso, o falante ouve a própria voz com um pitch diferente do usual ${ }^{(2)}$.

$\mathrm{O}$ fundamento subjacente é que, sob o efeito de um segundo sinal de fala, há a inibição, em nível central, do bloqueio neural involuntário, que se acredita ser o agente causal de todos os sintomas observáveis da gagueira. Quando apresentado um segundo sinal de fala, dependendo de sua sincronia linguística e temporal, a frequência da gagueira pode ser reduzida em aproximadamente $60 \%$. Esse segundo sinal pode ser entendido como uma "informação gestural" adicional que promove a fala fluente ${ }^{(3)}$.

Uma revisão sistemática consiste na aplicação de estratégias científicas que buscam a avaliação crítica e a síntese de um grande número de estudos sobre um determinado tópico. A relevância da realização de estudos dessa natureza é a capacidade de resumir e condensar resultados de vários estudos, produzindo diferentes indicadores quantitativos e qualitativos sobre o tópico pesquisado ${ }^{(4)}$.

\section{OBJETIVOS}

O objetivo desse estudo foi realizar uma revisão sistemática de pesquisas relacionadas aos efeitos das alterações dofeedback auditivo, especificamente do DAF e FAF, sobre a fluência da fala em pessoas com gagueira.

\section{ESTRATÉGIA DE PESQUISA}

Para o estabelecimento do método de pesquisa foram seguidos os preceitos do Cochrane Handbook ${ }^{(5,6)}$ :

1. Quanto à formulação da pergunta: analise dos textos sobre os efeitos das AFAs (DAF e FAF) sobre a fluência da fala em pessoas com gagueira, adultas, que tenham ou não sido submetidas a tratamentos anteriores para a gagueira;

2. Localização e seleção dos estudos: levantamento dos textos publicados sobre o assunto, sem delimitação temporal. Os artigos foram selecionados por meio da base de dados PubMed utilizando os descritores "altered auditory feedback and stuttering; delay auditory feedback and stuttering; frequency altered feedback and stuttering"; limitando-se a pesquisas realizadas com seres humanos, adultos, no idioma Inglês;

3. Compatibilização entre os pesquisadores: a busca de textos no banco de dados foi realizada independentemen- te pelos autores, visando minimizar possíveis perdas de citações.

\section{CRITÉRIOS DE SELEÇÃO}

Cada citação recuperada no banco de dados foi analisada independentemente pelos autores do estudo, que julgaram a pertinência de sua seleção e inclusão. Foram excluídas citações em línguas que não o Inglês. Citações que não permitiram o acesso ao texto completo (obtidos no Portal de Periódicos da CAPES), ou que foram repetidas por sobreposição das palavras chave também foram excluídas.

Dos textos completos obtidos foram excluídos: pesquisas realizadas exclusivamente com indivíduos fluentes; estudos de caso; revisões de literatura; cartas ao editor; e textos que não se relacionavam diretamente ao tema. Foram analisados os textos que efetivamente se relacionavam às pesquisas de tratamento com DAF e FAF.

Todas as etapas do estudo foram conduzidas independentemente pelos pesquisadores. Quando houve discordância, só foram incluídos os textos onde a posição final foi consensual. Pela natureza do estudo a pesquisa não foi passível de mascaramento.

\section{ANÁLISE DE DADOS}

1. Quanto aos indicadores da pesquisa foram considerados: objetivos; número e gênero dos participantes; faixa etária; variáveis de calibragem dos dispositivos; critérios de avaliação e controle dos tratamentos; resultados.

2. Quanto aos marcadores de qualidade dos estudos os textos foram analisados de acordo com: tipo de mascaramento; grupo controle; análise quantitativa dos dados; tratamento estatístico com confirmação da significância dos resultados; design da pesquisa.

\section{RESULTADOS}

Os dados mostram o percurso realizado para a seleção final dos textos analisados (Figura1). O percurso de seleção, dentro do método apresentado excluiu: citações cujo tema não era pertinente à pesquisa (19); textos de revisão de literatura (8); cartas ao editor (4); estudos de caso (3); estudos realizados exclusivamente com falantes fluentes (4); estudos realizados com portadores de gagueira neurogênica (2) e citações sem possibilidade de recuperação do texto na íntegra, fundamentalmente por serem textos antigos e não disponibilizados (37).

\section{Quanto aos objetivos}

Dos 24 textos analisados ${ }^{(7-30)}, 45,8 \%(n=11)$ tiveram como objetivo principal a avaliação da efetividade do dispositivo na redução da gagueira. Em 29,2\% (n=7) dos textos o objetivo principal foi o efeito do dispositivo (a curto, médio prazo e sobre a naturalidade) sobre a performance de fala. Outros objetivos compuseram $25 \%(n=6)$ das pesquisas tais como: modificações acústicas dos sons (ex: vogais) e de resposta auditiva (ex: uso mono ou binaural). 


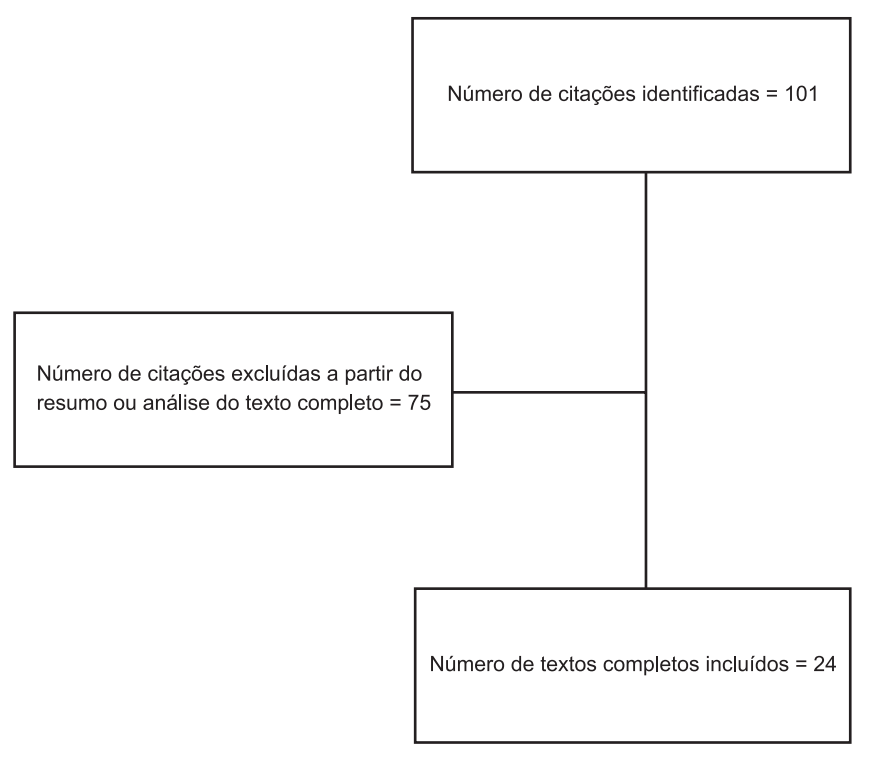

Figura 1. Percurso da busca realizada para seleção dos textos a serem analisados

\section{Quanto ao gênero e idade dos participantes}

Dos 24 textos analisados, $87,5 \%(\mathrm{n}=21)$ possuíam descrição do gênero dos participantes. A média do número de participantes com gagueira nos estudos foi de 12,3, com desvio-padrão 5,7 (Tabela 1). Houve, como esperado, um maior número de participantes do gênero masculino, numa proporção de 4,48/1.

Tabela 1. Análise do gênero

\begin{tabular}{lccc}
\hline & $\begin{array}{c}\text { Número de } \\
\text { gagos }\end{array}$ & $\begin{array}{c}\text { Sujeitos gênero } \\
\text { masculino }(\%)\end{array}$ & $\begin{array}{c}\text { Sujeitos gênero } \\
\text { feminino (\%) }\end{array}$ \\
\hline Média & 12,3 & 80,3 & 17,9 \\
DP & 5,7 & 16,9 & 16,5 \\
\hline
\end{tabular}

Legenda: $\mathrm{DP}=$ desvio-padrão

Quanto à variável idade dos participantes, apenas 19 textos especificavam a idade ou faixa etária. Das especificações disponíveis não foi possível calcular a média de idade. A faixa etária média dos estudos foi de 19 a 47 anos.

\section{Quanto às tarefas e variações na regulagem dos dispositivos}

Dos 24 textos analisados, houve uma distribuição balanceada entre as propostas de tarefas. As tarefas consideradas simples $(54,2 \%, \mathrm{n}=13)$ foram aquelas aplicadas nos estudos transversais e nos estudos que testaram uma única variável (ex: leitura de pequeno texto - monólogo ou ambos - com diferentes variações na calibragem do dispositivo ou diferentes dispositivos). As tarefas complexas $(45,8 \%, n=11)$ foram aquelas que controlaram mais de uma variável (ex: estudos longitudinais, estudos com juízes, tarefas competitivas, etc.).

Em 50\% $(n=12)$ dos textos analisados os pesquisadores contemplaram tarefas de fluência induzida (leitura, fala em coro, etc.) e fala espontânea (monólogo, conversação, etc.). Em
$20,8 \%$ dos estudos ( $\mathrm{n}=5)$ foi utilizada a leitura de pequenos textos; em 12,5\% (n=3) monólogo e conversação; em 12,5\% (n=3) outras atividades de fala (ex: telefone, fala automática, etc.); em $4,2 \%(n=1)$ outros tipos de leitura (palavras, sentenças, etc.).

Sobre as variações na regulagem dos dispositivos, diante da diversidade das calibrações apresentadas, optou-se pela divisão dos textos em dois grupos: textos que apresentaram AFA por utilização do DAF e textos que apresentaram AFA por utilização de FAF. Os textos que apresentavam aparelhos com a função COMBO (DAF associado ao FAF) foram pontuados em ambos os grupos pois se a análise fosse feita separadamente, esta seria praticamente individual, em virtude da grande variação de calibragem, causando uma grande dispersão dos dados.

Em relação aos estudos que utilizaram DAF $(n=19), 47,4 \%$ $(n=9)$ apresentaram uma calibração pontual do tempo de atraso e $52,6 \%(\mathrm{n}=10)$ apresentaram uma calibração do tempo de atraso variável. Dos estudos com DAF que apresentaram tempo de atraso pontual, 44,4\% $(\mathrm{n}=4)$ tiveram atraso de $60 \mathrm{~ms}, 33,3 \%$ $(\mathrm{n}=3)$ de $50 \mathrm{~ms}, 11,1 \%(\mathrm{n}=1)$ de $100 \mathrm{~ms}$ e $11,1 \%(\mathrm{n}=1)$ de 195 $\mathrm{ms}$. Dos estudos com DAF que apresentaram uma calibração do tempo de atraso variável, não foram encontrados estudos com a mesma variação, ou seja, dos 10 estudos analisados, cada um apresentou variação do tempo de atraso diferente dos outros. Nesses estudos, em $10 \%(\mathrm{n}=1)$ a calibração foi variável entre 55 a 105 ms; em 10\% (n=1) de 93 a 147 ms; em 10\% (n=1) de 30 a 120 ms; em $10 \%(n=1)$ de 30 a 60 ms; em $10 \%(n=1)$ de 60 a 90 ms; em $10 \%(n=1)$ de 90 a 250 ms; em $10 \%(n=1)$ de 50 a 75 ms; em 10\% (n=1) de 40 a 100 ms; em 10\% (n=1) de 100 a $200 \mathrm{~ms}$; em $10 \%(\mathrm{n}=1)$ de 55 a $100 \mathrm{~ms}$.

Em relação ao FAF, foi observada uma diferenciação na calibragem de frequência em virtude das especificações técnicas dos dispositivos utilizados. Alguns dispositivos apresentam variação de frequência em hertz (Hz), com incrementos de 500 $\mathrm{Hz}$ (positivos ou negativos, tendo uma variação de $-2 \mathrm{kHz}$ a +2 $\mathrm{kHz}$ ). Outros, apresentam a variação de frequência em escala de oitavas (em relação à frequência fundamental do paciente).

Entre os estudos analisados que utilizaram FAF $(\mathrm{n}=20), 55 \%$ $(\mathrm{n}=11)$ apresentaram uma calibração pontual da frequência e $45 \%(n=9)$ apresentaram uma calibração de frequência variável. Quanto aos estudos que apresentaram calibração pontual, $36,4 \%(\mathrm{n}=4)$ apresentaram variação de frequência de $+500 \mathrm{~Hz}$; $18,2 \%(n=2)$ de uma oitava abaixo em relação à frequência fundamental do paciente; $18,2 \%(\mathrm{n}=2)$ de meia oitava abaixo; $18,2 \%(\mathrm{n}=2)$ de um quarto de oitava acima; $9 \%(\mathrm{n}=1)$ de meia oitava acima. Já em estudos que apresentaram calibração de frequência variável, 33,3\% (n=3) apresentaram variação de um quarto de oitava abaixo a um quarto de oitava acima; $22,2 \%$ $(n=2)$ de meia oitava abaixo a meia oitava acima; $11,1 \%(n=1)$ de uma oitava abaixo a uma oitava acima; $11,1 \%(\mathrm{n}=1)$ de -2 $\mathrm{kHz}$ a $+2 \mathrm{kHz} ; 11,1 \%(\mathrm{n}=1)$ de $-500 \mathrm{~Hz}$ a $+500 \mathrm{~Hz} ; 11,1 \%$ $(\mathrm{n}=1)$ de $-500 \mathrm{~Hz} \mathrm{a}+1 \mathrm{kHz}$.

\section{Quanto aos resultados dos estudos}

Para a análise dos resultados obtidos nos 24 textos analisados, foram utilizados os seguintes critérios: positivo (quando a conclusão do estudo declarava que o dispositivo atingiu a meta 
proposta), negativo (quando a conclusão do estudo declarava que o dispositivo não atingiu a meta proposta) e inconclusivo (quando a conclusão do estudo indicava que o dispositivo foi eficiente em um aspecto e ineficiente em outro; quando o dispositivo se mostrou eficiente para parte do grupo e ineficiente para a outra parte; quando o dispositivo foi eficiente no pré-teste e ineficiente no seguimento). Para análise, foi usado o Teste Exato de Fisher com valor de $\mathrm{p} \leq 0,05$.

Tabela 2. Comparação entre eficiência e tipo de dispositivo utilizado

\begin{tabular}{lccc}
\hline & $\begin{array}{c}\text { Positivo } x \\
\text { Negativo }\end{array}$ & $\begin{array}{c}\text { Negativo } x \\
\text { Inconclusivo }\end{array}$ & $\begin{array}{c}\text { Positivo } x \\
\text { Inconclusivo }\end{array}$ \\
\hline DAF & 1,000 & 1,000 & 1,000 \\
FAF & $0,021^{*}$ & 0,462 & 0,286 \\
DAF+FAF & $<0,001^{*}$ & 0,590 & 0,100
\end{tabular}

* Valores significativos $(p \leq 0,05)-$ Teste Exato de Fisher

Legenda: $\mathrm{DAF}=$ delayed auditory feedback; FAF $=$ frequency-altered feedback

Em relação aos textos analisados, o uso exclusivo do DAF não apresentou resultados conclusivos. Não houve diferença entre resultados positivos, negativos, e inconclusivos. Para o FAF, houve diferença entre resultados positivos e negativos. Entretanto, não houve diferença quando comparados os resultados positivos e negativos com os inconclusivos. Para a combinação FAF+DAF houve diferença entre resultados positivos e negativos. Por outro lado, não houve diferença quando comparados os resultados positivos e negativos com os inconclusivos.

\section{Quanto aos marcadores de qualidade dos estudos}

Os textos foram analisados quanto ao tipo de mascaramento (simples/duplo cego, compatibilização entre juízes ou nenhum); grupo controle (existente ou não existente); análise quantitativa dos dados (sim ou não); tratamento estatístico que confirme a significância dos resultados (sim ou não); design da pesquisa (longitudinal ou transversal). Foi atribuído um ponto para cada um dos marcadores: simples/duplo cego ou compatibilização entre juízes; grupo controle existente; análise quantitativa dos dados; tratamento estatístico com confirmação da significância dos resultados; design de pesquisa longitudinal. Foi atribuída a pontuação zero para os demais marcadores. A qualificação dos estudos foi marcada na relação $5 / 5$ para os estudos melhor delineados e 0/5 para os estudos com maior fragilidade.

Em relação aos itens avaliados, $12,5 \%(\mathrm{n}=3)$ dos estudos apresentaram grupo controle; $37,5 \%(\mathrm{n}=9)$ contaram com algum tipo de mascaramento; $95,8 \%(n=23)$ tiveram algum tipo de análise quantitativa dos dados e 79,2 (n=19) aplicaram tratamento estatístico. Houve delineamento longitudinal em $28,8 \%(\mathrm{n}=5)$ das pesquisas.

Quanto à pontuação global, para os itens avaliados, nenhum estudo atingiu a pontuação plena. A pontuação de $4 / 5$ foi atingida em 20,8\% (n=5) dos textos e a pontuação de $3 / 5$ também foi atingida em $20,8 \%(\mathrm{n}=5)$ pesquisas. A pontuação $2 / 5$ foi alcançada por $45,8 \%(\mathrm{n}=11)$ dos estudos; $8,4 \%(\mathrm{n}=2)$ das pesquisas obtiveram a pontuação de $1 / 5$ e $4,1 \%(n=1)$ atingiram $0 / 5$.

\section{CONCLUSÃO}

Uma primeira informação sistemática tem como resultado que de um universo de 101 citações, 23,8\% dos textos publicados referem-se a pesquisas quantitativas, aplicadas às pessoas que gaguejam, com ou sem grupo controle, adultos, de ambos os gêneros, com ou sem tratamento. Não há textos similares em termos metodológicos, ou seja, não existem estudos que repliquem um determinado método em seu conjunto: perfil dos participantes, especificidade das tarefas e calibragem dos dispositivos.

Embora existam textos isoladamente consistentes, se observados os critérios de qualificação das pesquisas, nenhum estudo avaliado pode ser considerado plenamente satisfatório. Poucos estudos contemplam $80 \%$ dos requisitos para uma pesquisa consistente. Ainda não existem ensaios clínicos randomizados sobre o tema.

Dentro do paradigma que a revisão sistemática é a base para a prática clínica baseada em evidências, os resultados aqui obtidos indicam que o uso dos dispositivos de AFA na redução do número de eventos de gagueira ainda não tem suporte robusto em sua aplicabilidade. A variabilidade de método não permite uma resposta ou uma tendência de resposta que possa ser considerada consistente sobre a eficácia do dispositivo.

O uso exclusivo do DAF não apresenta resultados conclusivos, uma vez que resultados positivos, negativos, e inconclusivos são semelhantes entre os estudos analisados. Em relação ao uso do FAF há diferença na comparação de resultados positivos e negativos. Entretanto não há diferença quando comparados os resultados positivos e negativos com os inconclusivos. Para a combinação FAF+DAF há significância para resultados positivos quando comparados com os negativos, entretanto não há diferença significante quando comparados os resultados positivos com inconclusivos e negativos com inconclusivos.

Embora as limitações apontadas impeçam generalizações sobre a eficácia do uso do dispositivo para a redução da gagueira, essas mesmas limitações são recursos importantes para o planejamento de pesquisas futuras, em que deverão ser considerados principalmente: expansão do número de participantes, existência de grupo controle, mascaramento dos grupos, replicação das tarefas que se mostrem eficazes em seus propósitos e consistência na calibragem dos dispositivos.

\section{REFERÊNCIAS}

1. Stuart A, Kalinowski J. The perception of speech naturalness of posttherapeutic and altered auditory feedback speech of adults with mild and severe stuttering. Folia Phoniatr Logop. 2004;56(6):347-57.

2. Lincoln M, Packman A, Onslow M. Altered auditory feedback and the treatment of stuttering: a review. J Fluency Disord. 2006;31(2):71-89.

3. Guntupalli VK, Kalinowski J, Saltuklaroglu T, Nanjundeswaran C. The effects of temporal modification of second speech signals on stuttering inhibition at two speech rates in adults. Neurosci Lett. 2005;385(1):7-12.

4. Luna Filho B. A ciência e a arte de ler artigos médicos. São Paulo: Atheneu; 2010.

5. Higgins JPT, Green S, editors. Cochrane handbook for systematic reviews of interventions [Internet] Version 5.1.0 [updated March 2011; cited 2010 May 17]. [place unknown]: The Cochrane Collaboration; 2011. Available from www.cochrane-handbook.org. 
6. Hemingway P, Brereton N. What is a systematic review? [internet] 2009 [cited 2011 Jan 06]. (What is...? Series). Available from: http://www. whatisseries.co.uk/whatis/pdfs/What_is_syst_rev.pdf

7. Howell P. Changes in voice level caused by several forms of altered feedback in fluent speakers and stutterers. Lang Speech. 1990;33(Pt 4): 325-38.

8. Kalinowski J, Armson J, Roland-Mieszkowski M, Stuart A, Gracco VL. Effects of alterations in auditory feedback and speech rate on stuttering frequency. Lang Speech. 1993;36(Pt 1):1-16.

9. Macleod J, Kalinowski J, Stuart A, Armson J. Effect of single and combined altered auditory feedback on stuttering frequency at two speech rates. J Commun Disord. 1995;28(3):217-28.

10. Ingham RJ, Moglia RA, Frank P, Ingham JC, Cordes AK. Experimental investigation of the effects of frequency-altered auditory feedback on the speech of adults who stutter. J Speech Lang Hear Res. 1997;40(2):36172 .

11. Stuart A, Kalinowski J, Rastatter MP. Effect of monaural and binaural altered auditory feedback on stuttering frequency. J Acoust Soc Am. 1997;101(6):3806-9.

12. Armson J, Stuart A. Effect of extended exposure to frequency-altered feedback on stuttering during reading and monologue. J Speech Lang Hear Res. 1998;41(3):479-90.

13. Kalinowski J, Stuart A, Wamsley L, Rastatter MP. Effects of monitoring condition and frequency-altered feedback on stuttering frequency. $\mathrm{J}$ Speech Lang Hear Res. 1999;42(6):1347-54.

14. Natke U, GrosserKarl J, Kalveram T. Fluency, fundamental frequency, and speech rate under frequency-shifted auditory feedback in stuttering and nonstuttering persons. J Fluency Disord. 2001;26:227-41.

15. Sparks G, Grant DE, Millay K, Walker-Batson D, Hynan LS. The effect of fast speech rate on stuttering frequency during delayed auditory feedback. J Fluency Disord. 2002;27(3):187-200.

16. Van Borsel J, Reunes G, Van den Bergh N. Delayed auditory feedback in the treatment of stuttering: clients as consumers. Int J Lang Commun Disord. 2003;38(2):119-29.

17. Stager SV, Ludlow CL. The effects of fluency-evoking conditions on voicing onset types in persons who do and do not stutter. J Commun Disord. 1998;31(1):33-51.

18. Stuart A, Kalinowski J, Rastatter M, Saltuklaroglu T, Dayalu V. Investigations of the impact of altered auditory feedback in-the-ear devices on the speech of people who stutter: initial fitting and 4-month follow-up. Int J Lang Commun Disord. 2004;39(1):93-113.
19. Armson J, Kiefte M, Mason J, De Croos D. The effect of SpeechEasy on stuttering frequency in laboratory conditions. J Fluency Disord. 2006;31(2):137-52

20. Stuart A, Kalinowski J, Saltuklaroglu T, Guntupalli VK. Investigations of the impact of altered auditory feedback in-the-ear devices on the speech of people who stutter: one-year follow-up. Disabil Rehabil. 2006;28(12):757-65.

21. Lincoln M, Walker C. A survey of Australian adult users of altered auditory feedback devices for stuttering: use patterns, perceived effectiveness and satisfaction. Disabil Rehabil. 2007;29(19):1510-7.

22. O'Donnell JJ, Armson J, Kiefte M. The effectiveness of SpeechEasy during situations of daily living. J Fluency Disord. 2008;33(2):99-119.

23. Armson J, Kiefte M. The effect of SpeechEasy on stuttering frequency, speech rate, and speech naturalness. J Fluency Disord. 2008;33(2):12034.

24. Van Borsel J, Eeckhout H. The speech naturalness of people who stutter speaking under delayed auditory feedback as perceived by different groups of listeners. J Fluency Disord. 2008;33(3):241-51.

25. Antipova EA, Purdy SC, Blakeley M, Williams S. Effects of altered auditory feedback (AAF) on stuttering frequency during monologue speech production. J Fluency Disord. 2008;33(4):274-90.

26. Stuart A, Frazier CL, Kalinowski J, Vos PW. The effect of frequency altered feedback on stuttering duration and type. J Speech Lang Hear Res. 2008;51(4):889-97.

27. Kiefte M, Armson J. Dissecting choral speech: properties of the accompanist critical to stuttering reduction. J Commun Disord. 2008;41(1):33-48.

28. Pollard R, Ellis JB, Finan D, Ramig PR. Effects of the SpeechEasy on objective and perceived aspects of stuttering: a 6-month, phase I clinical trial in naturalistic environments. J Speech Lang Hear Res. 2009;52(2):516-33.

29. Saltuklaroglu T, Kalinowski J, Robbins M, Crawcour S, Bowers A. Comparisons of stuttering frequency during and after speech initiation in unaltered feedback, altered auditory feedback and choral speech conditions. Int J Lang Commun Disord. 2009;44(6):1000-17.

30. Lincoln M, Packman A, Onslow M, Jones M. An experimental investigation of the effect of altered auditory feedback on the conversational speech of adults who stutter. J Speech Lang Hear Res. 2010;53(5):1122-31. 